\title{
OTIOLTOMTS
}

Revista de economía, empresa y sociedad

Dosier sobre economía colaborativa (y II)

Nuevas estrategias y dimensiones alternativas de la economía de plataforma

Coordinador: Lluís Alfons Garay Tamajón

ALTERNATIVAS AL CAPITALISMO DE PLATAFORMA

\section{Democratizando la economía de plataforma}

\section{Melissa Renau Gano*}

Universitat Oberta de Catalunya

\section{Mayo Fuster Morell}

Universitat Oberta de Catalunya y Berkman Center

\section{Ricard Fspelt}

Universitat Oberta de Catalunya

RESUMEN Plataformas como Glovo, Deliveroo y UberEats dedicadas a la entrega a domicilio se han implantado en ciudades de todo el mundo. Su expansión ha ido acompañada de un impacto sobre los derechos de los trabajadores y trabajadoras, en lo que se considera capitalismo de plataforma. Aun así, existen alternativas democráticas vinculadas a la economía social y solidaria y a los comunes digitales. Este artículo analiza las calidades democráticas de cuatro plataformas alternativas de entrega a domicilio que tienen sede en Europa (CoopCycle, CILFé, Crow y Mensakas). Por un lado, los resultados muestran que estas plataformas tienen en consideración los derechos de sus trabajadores y trabajadoras y, por lo tanto, son una alternativa al capitalismo de plataforma. Por otro lado, se demuestra que estas plataformas tienen retos importantes, especialmente en relación con su sostenibilidad económica y escalabilidad.

PALABRAS CLAVE economía de plataforma; cooperativismo de plataforma; economía social; gig work; riders 


\title{
Democratising the platform economy
}

\begin{abstract}
Platforms such as Glovo, Deliveroo and Uber Eats that operate in the field of food delivery have set up in cities all around the world. The expansion of these platforms has had an impact on workers' rights in what is considered as platform capitalism. Even then, democratic alternatives connected to the social and solidarity economy and digital commons exist. This article analyses the democratic qualities of four alternative food delivery platforms with headquarters in Europe (CoopCycle, CILFé, Crow and Mensakas). On the one hand, the results show that these platforms consider their workers' rights; that is the main reason why they form an alternative to platform capitalism. On the other hand, the analysis shows that these platforms face important challenges, especially regarding their economic sustainability and scalability.
\end{abstract}

KEYWORDS platform economy; platform cooperativism; social economy; gig work; riders

\section{Introducción}

Al mismo tiempo que empresas como Glovo, Deliveroo y UberEats han atraído muchas inversiones, sus modelos de negocio son ampliamente cuestionados (Ball, 2020). Bajos salarios, malas condiciones laborales o carencia de protección social son características comunes de todas ellas (Urzì Brancati et al., 2020). A pesar de que estas plataformas difieren mucho en cuanto a los servicios que ofrecen, tienen en común que su modelo de negocio no sería posible sin la incorporación de sistemas de inteligencia artificial (IA) o dispositivos de geolocalización que permiten la recopilación masiva de datos (Lee et al., 2015; Ivanova et al., 2018; Veen et al., 2020). En definitiva, estas plataformas ejercen un gran control sobre sus trabajadores a través de la propia tecnología y no facilitan el acceso a los sistemas de protección social ni un desarrollo profesional (Forde et al., 2017; Parent-Thirion et al., 2017; Pesole et al., 2018; Kilhoffer et al., 2019). Así mismo estas plataformas también aceptan más trabajadores de los necesarios, cosa que exacerba todavía más la competencia por turnos y sirve como una herramienta más para disciplinar el trabajo (Altenried, 2019; Ivanova et al., 2018).

Estas prácticas que se aplican actualmente en la economía de plataforma gracias al apoyo de la tecnología (Lee et al., 2015; Rosenblat y Stark, 2015; Jarrahi y Sutherland, 2019) se basan en los principios del taylorismo (Taylor, 2006). Es decir, buscan mejorar la eficiencia económica y la productividad laboral implementando prácticas como incrementar el control sobre el proceso laboral, dividir las tareas en otras más simples y ofrecer un pago basado en el rendimiento de los trabajadores (McGaughey, 2018; Wood et al., 2019).

A pesar de que el debate público y la investigación se han centrado en estas plataformas extractivistas situadas bajo el paraguas del capitalismo de plataforma (Srnicek, 2016), existen plataformas realmente colaborativas basadas en la Economía Social y Solidaria (ESS) y el conocimiento abierto. De todos modos, hay pocos estudios basados en análisis de casos empíricos de plataformas alternativas o estos están centrados en proyectos paradigmáticos, como Fairbnb (Foramitti et al., 2020; Petruzzi et al., 2019) o SMart (Charles et al., 2020). Martinelli et al. (2019) estudiaron diferentes casos de cooperativas de plataforma, destacando su potencial para crear y promover puestos de trabajo de calidad mediante plataformas digitales, pero sin analizar en profundidad sus condiciones laborales o las calidades democráticas que las configuran.

El presente artículo pretende contribuir a este vacío proporcionando un análisis holístico de los elementos clave que permiten distinguir entre modelos de plataforma (Fuster Morell, 2018). En base al marco de las calidades democráticas de la economía de plataforma (figura 1), proponemos un análisis holístico de los elementos clave que constituyen una plataforma y su impacto (Fuster Morell y Espelt, 2019). Para hacerlo hemos hecho una et- 
nografía digital de cuatro plataformas de entrega a domicilio y una entrevista semiestructurada a cada uno de los casos. La recogida de datos se llevó a cabo entre julio y diciembre de 2019.

\section{Calidades democráticas de las plataformas digitales}

El marco de las calidades de sostenibilidad de la economía de plataforma (figura 1) se compone de seis dimensiones que permiten un análisis holístico de los modelos de plataforma. La dimensión de gobernanza evalúa la gobernanza en cuanto a la provisión de plataformas (transparencia, políticas de participación y constitución legal) y la gobernanza en cuanto a la interacción de la plataforma (haciendo coincidir las funcionalidades de la plataforma con el grado en que los usuarios pueden participar). La dimensión del modelo económico estudia la relación entre los beneficios económicos (distribución y destino) y su impacto social (condiciones y derechos laborales, tipos de crecimiento) y el modelo de sostenibilidad económica del proyecto. Las políticas tecnológicas hacen referencia a la arquitectura y el software tecnológico teniendo en cuenta el grado de reproductibilidad, determinado por el tipo de licencia. Se consideran dos elementos de las políticas de conocimiento: el acceso a los datos generados y la licencia del contenido. Finalmente, la dimensión de responsabilidad social e impacto evalúa las implicaciones generadas por la actividad de la plataforma (exclusión, desigualdad de género, impacto ambiental, etc.).

Figura 1. Marco de calidades democráticas de la economía de plataforma

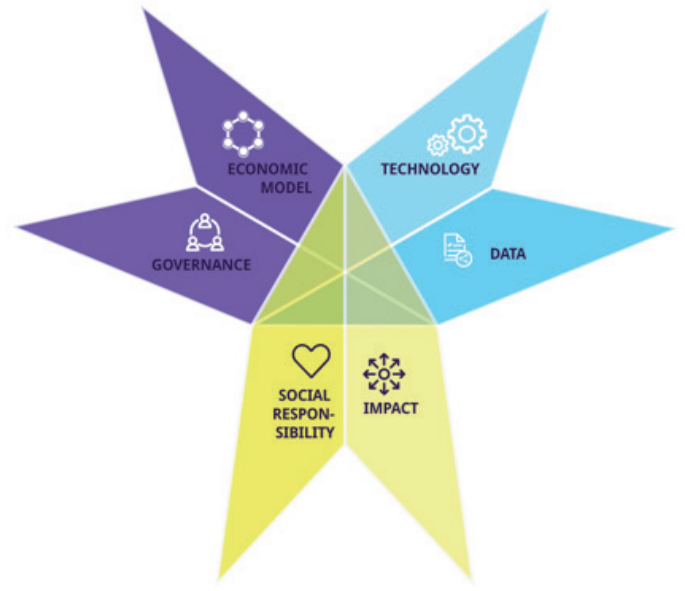

\section{Modelos de economía de plataforma}

El marco de las calidades democráticas de las plataformas digitales parte de la hipótesis de que es posible distinguir entre tres modelos de plataforma diferentes: unicornios, open commons y platform coops. En esta sección, se presentan y analizan los tres modelos y se relacionan con el marco de calidades democráticas presentado en la sección previa.

La producción entre iguales basada en los comunes (Benkler, 2006) es un concepto utilizado para describir formas de producción caracterizadas por la apertura a la participación (Fuster Morell, 2010), una fuerte igualdad en la distribución de las contribuciones a la comunidad (Ortega, 2009), descentralización (Crowston y Howison, 2006; Lanzara y Morner, 2004), modularidad y granularidad (Benkler, 2006), no coercitividad y una coordinación basada en la estigmergia (Siefkes, 2010), procesos transparentes (Bauwens, 2007), propiedad intelectual comunitaria (Wark, 2004) y las dimensiones del valor más allá de las concepciones monetarias (Fuster Morell et al., 2016). 
Las plataformas open commons son generalmente promovidas por fundaciones de gobierno comunitarias. Este modelo de plataforma utiliza el software libre de código abierto (FLOSS) y licencias abiertas que abren datos y conocimientos, lo que favorece la economía circular, atiende al impacto ambiental y se orienta al valor social.

Las unicornio hacen referencia a las startups de plataformas promovidas por empresas tecnológicas con una alta capitalización del mercado. El término «unicornio» fue utilizado por primera vez por Aileen Lee (2013) en una publicación en TechCrunch que hacía referencia a empresas nacidas después del 2003 y que se habían valorado en hasta 1.000 millones de dólares de capitalización bursátil en algún momento de su ciclo de vida. Las unicornio son empresas multinacionales orientadas exclusivamente al beneficio económico y que restringen enormemente su gobernanza. Normalmente se desarrollan con software propietario y datos cerrados. Las unicornio producen impactos disruptivos sobre la soberanía de las ciudades, el medio ambiente, la inclusión y el género.

Scholz y Schneider popularizaron el término "cooperativismo de plataforma» (Scholz, 2016; Scholz y Schneider, 2017). Según Scholz (2016), las plataformas se tienen que basar en los valores del cooperativismo y las herramientas digitales tienen que aumentar la escalabilidad de las plataformas y su impacto social y económico. Al mismo tiempo, Fuster Morell (2017) afirmó que las cooperativas de plataforma tendrían que adoptar programas y licencias abiertas teniendo en cuenta la importancia de su arquitectura tecnológica.

\section{Cuatro plataformas democráticas de ámbito europeo}

A partir del marco analítico presentado en la sección 2, procedemos al estudio de cuatro casos de plataformas democráticas de entrega de alimentos con presencia en la Unión Europea, para analizar su caracterización.

\subsection{CoopCycle}

CoopCycle es una federación de cooperativas creada el 2017 en París con el objetivo de reducir los gastos gracias a la agrupación de recursos y, al mismo tiempo, fortalecer el poder de negociación de los derechos de los trabajadores. A pesar de que el grosor de actividad está en Francia, también tiene entidades en Canadá, Reino Unido, Alemania, Bélgica, Italia, Suecia, Polonia y España y está desarrollando nuevos nodos en Sudamérica y al este de Europa.

- Gobernanza: para asegurar una base democrática, todos los miembros locales de la federación de CoopCycle tienen que ofrecer contratos asalariados a sus mensajeros tan pronto como puedan y organizar democráticamente la distribución de los ingresos. A nivel federativo, las cooperativas locales deciden juntas, en base a una cooperativa, una voz, sobre cuestiones clave como el nivel de contribución de cada miembro al fondo mutuo según sus ingresos, el destino de este fondo o el régimen salarial por los servicios prestados.

- Modelo económico: todavía no se ha conseguido un modelo de sostenibilidad económica, pero, cuando se alcance, los beneficios se quieren reinvertir en el proyecto. CoopCycle tiene un modelo de crecimiento federado y con una descentralización progresiva de la gobernanza de la plataforma. Actualmente, cada cooperativa local mutualiza según sus ingresos/recursos ( $2 \%$ de su valor añadido) y se puede beneficiar de todos los servicios de CoopCycle.

- Políticas de conocimiento y datos: CoopCycle permite el acceso libre al código de la plataforma a las empresas con una base ética sólida a través de una licencia copyleft. Es decir, entidades que tienen como principio garantizar los derechos de los trabajadores y la gestión democrática interna. En cuanto a los datos, la plataforma solo recopila la información necesaria para completar la entrega (nombre, correo electrónico y teléfono).

- Responsabilidad social: solo pueden adherirse a la federación las cooperativas que tengan un gasto carbono cero (a través del uso de bicicletas mecánicas o eléctricas) y que tengan previsto un modelo de empleo de mensajeros asalariado. 


\subsection{La Cour Cyclette (CILFé)}

El servicio de entrega de bicicletas de La Cour Cyclette es una empresa cooperativa que opera en la Val de Marne, al sudeste de la ciudad de París. El servicio de entrega de bicicletas de La Cour Cyclette tiene como objetivo promover el desarrollo de empresas locales combatiendo la degradación de las condiciones laborales.

- Gobernanza: la organización cooperativa promueve que cada miembro tenga derecho a voto en la toma de decisiones. La remuneración y las condiciones laborales son acordadas conjuntamente por los socios cooperativos. A nivel tecnológico, La Cour Cyclette no utiliza sistemas de gestión algorítmica de control de los trabajadores.

- Modelo económico: como plataforma cooperativa, los beneficios se reinvierten en el proyecto. La Cour Cyclette es la única entidad de este estudio que ha logrado un modelo de sostenibilidad económica sólido, a través de colaboraciones con la administración pública y cuotas de socio.

- Política tecnológica: uso del software CoopCycle.

- Políticas de conocimiento y datos: no se pueden acceder a los datos generados en ningún formato.

- Responsabilidad social: La Cour Cyclette sitúa los valores de la economía circular en el centro de su estrategia. Aparte de reducir las emisiones en la entrega de la última milla, proporcionan materiales educativos para concienciar sobre el consumo sostenible.

\subsection{Crow Cycle Courier Collective (Crow)}

Crow es una cooperativa de mensajería de bicicletas independiente que opera en Berlín y se basa en la autogestión y la economía circular.

- Gobernanza: organización cooperativa democrática de quince miembros, diez hombres y cinco mujeres. A pesar de que actualmente los mensajeros de la plataforma son autónomos, la empresa tiene un plan para evitar la degradación de las condiciones laborales. De hecho, el reconocimiento jurídico de autónomo se acordó entre los miembros, valorando su situación económica y sus necesidades.

- Modelo económico: cada trabajador recibe un mínimo garantizado por hora. Esto hace que a menudo los sueldos sean mejores que cualquier otra empresa de mensajería de Berlín. El modelo de crecimiento se basa en aumentar el número de miembros de la cooperativa sin modificar la estructura de gobierno.

- Política tecnológica: uso del software de CoopCycle.

- Políticas de conocimiento y datos: no es posible exportar, copiar ni acceder a los datos.

- Responsabilidad social: Crow promueve el reciclaje y la circularidad de los materiales ofreciendo un tipo de servicio que mejora la eficiencia energética. También dan prioridad a la responsabilidad social cuando escogen los proveedores de los servicios que requieren.

\subsection{Mensakas}

Mensakas fue fundada en 2018 en Barcelona por exmensajeros de Glovo y Deliveroo. Su misión es demostrar que es posible crear una plataforma de distribución responsable.

- Gobernanza: cooperativa democrática formada por ocho trabajadores, cinco hombres y tres mujeres. Todas las personas son empleados directos y ganan el salario mínimo. Por decisión de la cooperativa, las mujeres tienen un $5 \%$ más de salario que los hombres.

- Modelo económico: los beneficios de la entidad se reinvierten en el proyecto. Su modelo de crecimiento se basa en la reproducción del proyecto y la descentralización progresiva de la gobernanza de la plataforma. De hecho, ayudan a iniciativas de otras ciudades que tienen como objetivo ofrecer una alternativa a las grandes plataformas globales como Deliveroo o Glovo. Mensakas ha logrado la sostenibilidad económica durante el 2020, a través de fondos públicos, las cuotas obligatorias de los miembros y los beneficios de su propia actividad. 
- Política tecnológica: actualmente utilizan el software de CoopCycle, pero tienen previsto desarrollar su propia aplicación que incorpore algunas necesidades específicas.

- Políticas de conocimiento y datos: no es posible exportar, copiar ni acceder a los datos.

- Responsabilidad social: Mensakas promueve el reciclaje y la circularidad de los materiales. Cuando hayan desarrollado su propia aplicación tienen previsto alojarla en servidores de energía verde. La entidad da prioridad a la responsabilidad social cuando eligen los proveedores de servicios. También tienen un plan de formación interno que permite fomentar el conocimiento compartido.

La tabla comparativa de los cuatro casos (tabla 1) permite observar que, a pesar de que no hay ningún caso que cumpla al 100\% las calidades democráticas en las cinco dimensiones definidas, todas las plataformas tienen un buen nivel de desempeño.

Tabla 1. Tabla comparativa entre casos analizados. Verde: desempeño; naranja: desempeño parcial; rojo: no desempeño.

\begin{tabular}{|l|l|l|l|l|l|}
\hline \multicolumn{1}{|c|}{ Dimensiones } & \multicolumn{1}{|c|}{ Subdimensiones } & CoopCycle & CILFé & Crow & Mensakas \\
\hline Gobernanza & Formato jurídico & & & \\
\hline & Participación & & & \\
\hline Modelo económico & Misión & & & \\
\hline & Modelo de crecimiento & & & & \\
\hline & Sostenibilidad & & & \\
\hline & Financiación no lucrativa & & & & \\
\hline Políticas tecnológicas & Software abierto & & & & \\
\hline & Descentralización & & & \\
\hline Políticas de conocimiento & Licencia copyleft & & & \\
\hline & Datos abiertos & & & \\
\hline Responsabilidad social & Inclusión socioeconómica & & & & \\
\hline & Sensibilidad ambiental & & & & \\
\hline
\end{tabular}

\section{Conclusiones}

Las condiciones laborales vinculadas a la economía de plataforma son vanguardia del debate público y la agenda de investigación porque empresas como Glovo, Deliveroo y UberEats provocan una degradación de las condiciones laborales y vulneran los derechos de sus trabajadores (Tassinari y Maccarrone, 2020; Wood et al., 2019). En paralelo y a pesar de tener menos atención mediática y de investigación, la economía de plataforma también ofrece diversidad de modelos de negocio que son afines a las calidades democráticas que emergen de los principios de la economía social y solidaria y los comunes digitales (Fuster Morell, 2018). Este 
artículo se centra en el estudio de cuatro plataformas alternativas del ámbito de la entrega a domicilio con sede en Europa que se analizan a través del marco de calidades democráticas de las plataformas digitales (Fuster Morell y Espelt, 2019).

El resultado del análisis señala que las cuatro plataformas estudiadas (CoopCycle, CILFé, Crow, Mensakas) se muestran como una alternativa al capitalismo de plataforma (Srnicek, 2016) y fomentan alternativas democráticas de economía de plataforma. Sin embargo, el estudio detallado de estas iniciativas muestra que tienen retos importantes.

La gobernanza es el elemento más destacado de todas ellas. No solo porque las cuatro entidades estudiadas tienen forma jurídica cooperativa, sino porque fomentan la participación horizontal y democrática de sus miembros. Respecto al modelo económico, todas las organizaciones estudiadas tienen un enfoque sin ánimo de lucro. De hecho, la mayoría reinvierten o tienen la intención de reinvertir beneficios en su proyecto. Además, el modelo de crecimiento se basa en la reproductibilidad y el crecimiento sin cambios en la misión del proyecto. Es decir, evitando modelos económicos especulativos. Aun así, el reto más importante de estas cuatro organizaciones es la sostenibilidad económica. A pesar de que La Cour Cyclette (CILFé) y, recientemente, Mensakas han llegado a un punto de equilibrio entre ganancias y gastos, se evidencia que la sostenibilidad económica es uno de los principales retos del cooperativismo de plataforma y de los proyectos open commons.

En términos de políticas tecnológicas, todos los casos promueven software abierto y arquitecturas tecnológicas descentralizadas. En cambio, las políticas de conocimiento y datos no contemplan los datos abiertos. Por lo tanto, a pesar de que el software sea libre y con licencia copyleft, no es posible descargar los datos generados por los usuarios.

Finalmente, la práctica totalidad de los proyectos cuidan su responsabilidad social y medioambiental, priorizando los proveedores de Economía Social y Solidaria y promoviendo un consumo responsable, y son conscientes del impacto ambiental de la infraestructura de la plataforma, aunque todavía no utilicen servidores ecológicos eficientes.

Estos hallazgos contribuyen al conocimiento emergente de literatura académica y divulgativa destinada a comprender estas alternativas de economía de plataforma, especialmente a través del análisis empírico de casos. Así mismo, esta investigación demuestra que están surgiendo organizaciones democráticas alternativas a las plataformas unicornio y, a pesar de que presentan retos importantes en cuanto a la sostenibilidad y la escalabilidad económica, prevén un futuro alternativo en que la tecnología no se utiliza como excusa para un mayor control del puesto de trabajo, sino como una herramienta para promover organizaciones más horizontales, democráticas y que protegen los derechos laborales de sus trabajadores.

\section{Bibliografía}

ALTENRIED, Moritz. On the last mile: Logistical urbanism and the transformation of labour. En: Work Organisation, Labour \& Globalisation, 2019. Vol. 13, núm. 1, págs. 114-129. DOl: https://doi.org/10.13169/workorgalaboglob.13.1.0114.

BALL, James. Deliveroo was the poster child for venture capitalism. It's not looking so good now. En: The Guardian, 2 de mayo de 2020 [en línea]. Disponible en: https://www.theguardian.com/commentisfree/2020/ may/02/deliveroo-venture-capitalism-food-delivery-business-model.

BAUWENS, Michel. Peer-to-Peer Governance, Production and Property: P2P as a Way of Living. Part 1. En: Master New Media, 20 de octubre de 2007 [en línea]. Disponible en: https://communities-dominate.blogs.com/ brands/2007/10/peer-to-peer-go.html.

BENKLER, Yochai. The wealth of networks: How social production transforms markets and freedom. Yale University Press, 2006.

CHARLES, Julien, FERRERAS, Isabelle y LAMINE, Auriane. A freelancers' cooperative as a case of democratic institutional experimentation for better work: A case study of SMart-Belgium. En: Transfer: European Review of Labour and Research, 2020. Vol. 26, núm. 2, págs. 157-174. DOl: https://doi.org/10.1177/1024258920919686. 
CROWSTON, Kevin y HOWISON, James. Hierarchy and centralization in free and open source software team communications. En: Knowledge, Technology \& Policy, 2006. Vol. 18, núm. 4, págs. 65-85. DOI: https://doi.org/10.1007/s12130-006-1004-8.

FORAMITTI, Joël, VARVAROUSIS, Angelos y KALLIS, Giorgos. Transition within a transition: How cooperative platforms want to change the sharing economy. En: Sustainability Science, 2020. Vol. 15, núm. 4, págs. 1185-1197. DOI: https://doi.org/10.1007/s11625-020-00804-y.

FORDE, Chris et al. The Social Protection of Workers in the Platform Economy. Policy Department A: Economic and Scientific Policy - European Parliament, 2017. Pág. 128 [en línea]. Disponible en: https://www.europarl. europa.eu/thinktank/en/document.html?reference=IPOL_STU(2017)614184.

FUSTER MORELL, Mayo. Participation in online creation communities: Ecosystemic participation? En: Conference Proceedings of JITP 2010: The Politics of Open Source, 2010. Vol. 1, págs. 270-295. Disponible en: https://scholarworks.umass.edu/jitpc2010/1/.

FUSTER MORELL, Mayo. Towards a Theory of Value of Platform Cooperativism. En: Scholz Trebor y Schneider Nathan (eds.), Ours to hack and to own: The rise of platform cooperativism, a new vision for the future of work and a fairer internet. OR Books, 2017.

FUSTER MORELL, Mayo (ed.). Sharing cities: A worldwide cities overview on platform economy policies with a focus on Barcelona. Sehen, Editorial UOC, 2018. Disponible en: http://www.sharingcitiesaction.net/wp-content/ uploads/2018/11/SharingCities_book.pdf.

FUSTER MORELL, Mayo y ESPELT, Ricart. A Framework to Assess the Sustainability of Platform Economy: The Case of Barcelona Ecosystem. En: Sustainability, 2019. Vol. 11, núm. 22, págs. 6450. DOI: https://doi.org/10.3390/su11226450.

FUSTER MORELL, Mayo, SALCEDO, Jorge y BERLINGUER, Marco. Debate About the Concept of Value in Commons-Based Peer Production. En: BAGNOLI, Franco et al. (eds.), Internet Science, vol. 9934, págs. 27-41. Springer International Publishing, 2016. DOI: https://doi.org/10.1007/978-3-319-45982-0_3.

IVANOVA, Mirela et al. The App as a Boss? Control and Autonomy in Application-Based Management. EuropaUniversität Viadrina, 2018. Pág. 27. Disponible en: https://opus4.kobv.de/opus4-euv/frontdoor/index/index/ docld/388.

JARRAHI, Mohammad y SUTHERLAND, Will. Algorithmic Management and Algorithmic Competencies: Understanding and Appropriating Algorithms in Gig Work. En: TAYLOR, C. et al. (eds.), Information in Contemporary Society, vol. 11420, págs. 578-589. Springer International Publishing, 2019. DOI: https://doi.org/10.1007/9783-030-15742-5_55.

KILHOFFER, Zachary et al. Study to gather evidence on the working conditions of platform workers. Luxemburgo, Publications Office of the European Union, 2019. Disponible en: https://ec.europa.eu/social/main.jsp?catld=8 9\&furtherNews=yes\&langld=en\&newsld=9582.

LANZARA, G. y MORNER, Michèle. Making and sharing knowledge at electronic crossroads: The evolutionary ecology of open source. Fifth European Conference on Organizational Knowledge, Learning and Capabilities, Innsbruck, Austria, 2004.

LEE, Min Kyung et al. Working with Machines: The Impact of Algorithmic and Data-Driven Management on Human Workers. En: Proceedings of the 33rd Annual ACM Conference on Human Factors in Computing Systems CHI '15, 2015. Págs. 1603-1612. DOI: https://doi.org/10.1145/2702123.2702548.

LEE, Aileen. Welcome To the unicorn club: Learning from billion-dollar startups. En: Techcrunch, 2 de noviembre de 2013 [en línea]. Disponible en: https://techcrunch.com/2013/11/02/welcome-to-the-unicorn-club/.

MARTINELLI, Francesca. Platform Cooperativism in Italy and in Europe. CIRIEC International, 2019.

MCGAUGHEY, Ewan. Taylorooism: When network technology meets corporate power: Technology meets corporate power. En: Industrial Relations Journal, 2018. Vol. 49, núm. 5-6, págs. 459-472. DOI: https://doi. org/10.1111/irj.12228.

ORTEGA, Felipe. Wikipedia: A Quantitative Analysis. Universidad Rey Juan Carlos, 2009.

PARENT-THIRION, Agnès. et al. 6th European Working Conditions Survey: Overview report (2017 update). Luxemburgo, Publications Office of the European Union, 2017. 
PESOLE, A. et al. Platform workers in Europe evidence from the COLLEEM survey. Luxemburgo, Publications Office of the European Union, 2018. Pág. 65.

PETRUZZI, Marina A., SHEPPARD, Valerie y MARQUES, Catarina. Positioning Airbnb and Fairbnb in the sharing-exchange continuum. En: Current Issues in Tourism, 2019. DOl: https://doi.org/10.1080/13683500.2019.1697650.

ROSENBLAT, A. y STARK, L. Uber's Drivers: Information Asymmetries and Control in Dynamic Work. SSRN Electronic Journal, 2015. DOl: https://doi.org/10.2139/ssrn.2686227.

SCHLOZ, Trebor. Platform cooperativism. Challenging the Corporate Sharing Economy. En: Rosa Luxemburg Stiftung, 2016 [en línea]. Disponible en: http://www.rosalux-nyc.org/platform-cooperativism-2/.

SCHOLZ, Trebor y SCHNEIDER, Nathan (eds.). Ours to hack and to own: The rise of platform cooperativism, a new vision for the future of work and a fairer internet. OR Books, 2017.

SIEFKES, Christian. Self-organized Plenty. En: Keimform. Auf Der Suche Nach Dem Neuen Im Alten, 23 de noviembre de 2010 [en línea]. Disponible en: https://keimform.de/2010/self-organized-plenty/.

SRNICEK, Nick. Platform capitalism. Polity Press, 2016.

TASSINARI, Arianna y MACCARRONE, Vicenzo. Riders on the Storm: Workplace Solidarity among Gig Economy Couriers in Italy and the UK. En: Work, Employment and Society, 2020. Vol. 34, núm. 1, págs. 35-54. https://doi.org/10.1177/0950017019862954.

TAYLOR, Frederik. The Principles of Scientific Management. Cosimo, Inc, 2006.

URZI BRANCATI, M.C., PESOLE, A., FERNÁNDEZ-MACÍAS, E. New evidence on platform workers in Europe results from the second COLLEEM survey. Luxemburgo, Publications Office of the European Union, 2020. DOI: https://doi.org/10.2760/459278.

VEEN, Alex, BARRATT, Tom y GOODS, Caleb. Platform-Capital's 'App-etite' for Control: A Labour Process Analysis of Food-Delivery Work in Australia. En: Work, Employment and Society, 2020. Vol. 34, núm. 3, págs. 388-406. DOI: https://doi.org/10.1177/0950017019836911.

WARK, McKenzie. A hacker manifesto. Harvard University Press, 2004.

WOOD, Alex et al. Good Gig, Bad Gig: Autonomy and Algorithmic Control in the Global Gig Economy. En: Work, Employment and Society, 2019. Vol. 33, núm. 1, págs. 56-75. DOl: https://doi.org/10.1177/0950017018785616.

Cita recomendada: RENAU*, Melissa; FUSTER, Mayo; ESPELT, Ricard. Alternativas al capitalismo de plataforma. Democratizando la economía de plataforma. Oikonomics [en línea]. Mayo 2021, n. 15. ISSN: 2339-9546. DOI: https://doi.org/10.7238/o.n15.2102

Los textos publicados en esta revista están sujetos -si no se indica lo contrario- a una licencia de Reconocimiento 4.0 Internacional de Creative Commons. Puede copiarlos, distribuirlos, comunicarlos públicamente, hacer obras derivadas siempre que reconozca los créditos de las obras (autoría, nombre de la revista, institución editora) de la manera especificada por los autores o por la revista. La licencia completa se puede consultar en https://creativecommons.org/licenses/by/4.0/deed.es_ES.

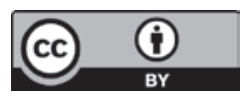




\section{Melissa Renau Gano \\ mrenauc@uoc.edu \\ Universitat Oberta de Catalunya}

Melissa Renau es doctoranda en Dimmons en el Internet Interdisciplinary Institute de

la Universitat Oberta de Catalunya. Actualmente desarrolla su tesis doctoral en Dimmons como parte del proyecto europeo Platform Labour in Urban Spaces sobre la flexibilidad y las condiciones laborales en la economía de plataformas. Tiene un máster en Sociología por la Universitat de Barcelona y una licenciatura en Administración de Empresas por la Universitat Pompeu Fabra.

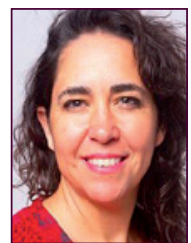

\section{Mayo Fuster Morell}

mfuster@uoc.edu

\section{Universitat Oberta de Catalunya y Berkman Center}

Mayo Fuster Morell es investigadora principal de Dimmons en el Internet Interdisciplinary Institute de la Universitat Oberta de Catalunya. Profesora afiliada al Berkman Klein Center for Internet and Society de la Universidad de Harvard. Es directora de la Cátedra Barcelona UOC en Economía Digital: para una economía colaborativa centrada en el bienestar de las personas y el derecho a la ciudad, de la UOC junto al Ayuntamiento de Barcelona y Barcelona Activa. Ha sido la investigadora principal de la UOC para los proyectos europeos DECODE: Decentralised Citizens Owned Data Ecosystem y PLUS: Platform Labor in Urban Spaces. Fue la investigadora principal del proyecto europeo P2Pvalue: Techno-social platform for sustainable models and value generation in commons-based peer production in the Future Internet. El 2010, concluyó su tesis doctoral en el Instituto Universitario Europeo de Florencia sobre la gobernanza de la producción procomún entre iguales y cuenta con numerosas publicaciones en este campo.

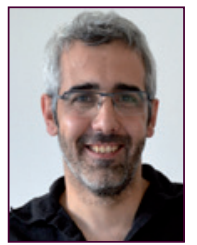

\section{Ricard Fspelt ricardespelt@uoc.edu Universitat Oberta de Catalunya}

Investigador senior y coordinador del equipo de investigación Dimmons en el Internet Interdisciplinary Institute de la Universitat Oberta de Catalunya. Centró su tesis doctoral en estudiar el impacto de Internet en el ámbito del cooperativismo agroecológico y ha desarrollado su línea de investigación en torno al cooperativismo de plataforma. Es licenciado en Bellas Artes y tiene una larga trayectoria en el eje que interrelaciona la educación y el arte. Es investigador posdoctoral y coordinador del proyecto europeo PLUS: Platform Labor in Urban Spaces y fue coordinador del proyecto DECODE: Decentralised Citizens Owned Data Ecosystem en Dimmons. 